https://helda.helsinki.fi

\title{
The CRCbiome study : a large prospective cohort study examining the role of lifestyle and the gut microbiome in colorectal cancer screening participants
}

\section{Kvaerner, Ane Sorlie}

2021-08-18

Kvaerner, A S , Birkeland, E, Bucher-Johannessen, C, Vinberg, E, Nordby , J I , Kangas , H, Bemanian, V , Ellonen, P, Botteri , E, Natvig , E, Rognes , T, Hovig , E, Lyle , R , Ambur, O H , de Vos , W M , Bultman , S, Hjartaker , A, Landberg , R, Song , M , Blix , H S , Ursin , G, Randel , K R , de Lange , T , Hoff , G , Holme , O, Berstad , P \& Rounge , T B 2021, ' The CRCbiome study : a large prospective cohort study examining the role of lifestyle and the gut microbiome in colorectal cancer screening participants ' , BMC Cancer , vol. 21 , no. 1 , 930 . https://doi.org/10.1186/s12885-021-08640-8

http://hdl.handle.net/10138/335036

https://doi.org/10.1186/s12885-021-08640-8

cc_by

publishedVersion

Downloaded from Helda, University of Helsinki institutional repository.

This is an electronic reprint of the original article.

This reprint may differ from the original in pagination and typographic detail.

Please cite the original version. 


\section{The CRCbiome study: a large prospective cohort study examining the role of lifestyle and the gut microbiome in colorectal cancer screening participants}

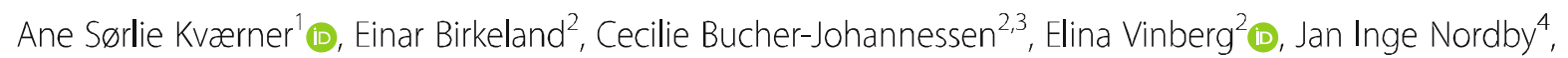
Harri Kangas ${ }^{5}$, Vahid Bemanian ${ }^{6}$, Pekka Ellonen ${ }^{5}$ (D) Edoardo Botteri ${ }^{1,2}$ (D), Erik Natvig ${ }^{1}$, Torbjørn Rognes ${ }^{7,8}$ (D), Eivind Hovig ${ }^{3,7} \mathbb{B}$, Robert Lyle ${ }^{9,10}$, Ole Herman Ambur ${ }^{11,12} \mathbb{B}$, Willem M. de $\operatorname{Vos}^{13,14} \mathbb{B}$, Scott Bultman ${ }^{15}$, Anette Hjartåker ${ }^{16}$, Rikard Landberg ${ }^{17}$ (D) Mingyang Song ${ }^{18,19,20}$ (D) , Hege Salvesen Blix $21,22 \mathbb{C}$, Giske Ursin ${ }^{23}$ (D), Kristin Ranheim Randel', Thomas de Lange $e^{24,25,26} \mathbb{D}$, Geir Hoff ${ }^{1,27}$ (D), Øyvind Holme $e^{1,28,29}$, Paula Berstad ${ }^{1 *+} \mathbb{D}$ and Trine B. Rounge $\mathrm{e}^{2,7^{*+}}$ (1)

\footnotetext{
Abstract

Background: Colorectal cancer (CRC) screening reduces CRC incidence and mortality. However, current screening methods are either hampered by invasiveness or suboptimal performance, limiting their effectiveness as primary screening methods. To aid in the development of a non-invasive screening test with improved sensitivity and specificity, we have initiated a prospective biomarker study (CRCbiome), nested within a large randomized CRC screening trial in Norway. We aim to develop a microbiome-based classification algorithm to identify advanced colorectal lesions in
} 




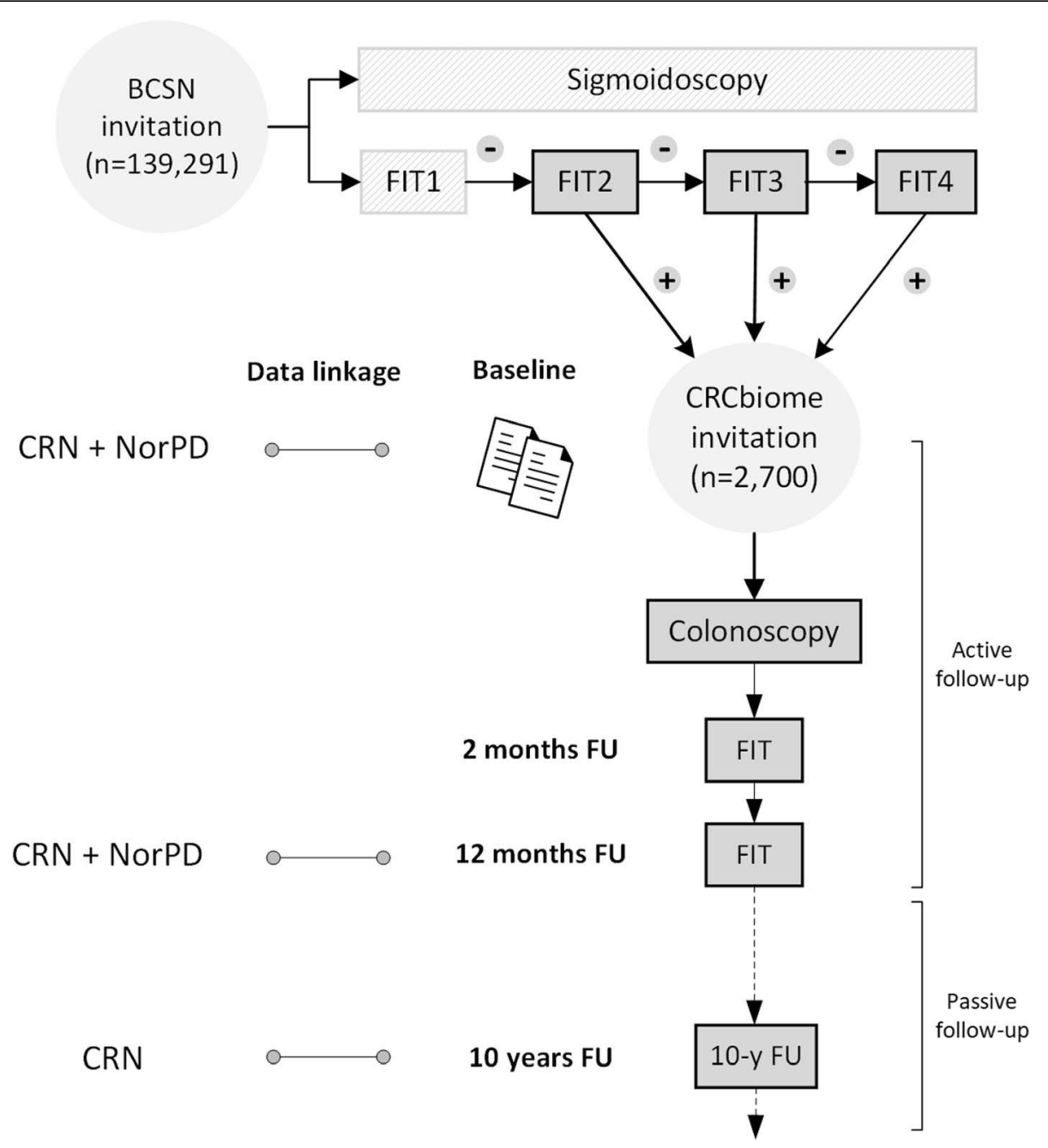

Fig. 1 Flowchart of the CRCbiome study, nested within the BCSN. Abbreviations: BCSN, Bowel Cancer Screening in Norway; CRN, Cancer Registry of Norway; FIT, fecal immunochemical test; FU, follow-up; NorPD, Norwegian Prescription Database

CRCbiome study. Recruitment for the CRCbiome study started in 2017, and will continue until a minimum of 2700 participants have been invited. So far, 2426 have been invited and 1413 (58\%) have agreed to participate. With the current participation rate, we expect recruitment to be completed by March 2021 with a final number of participants of about 1600 (see below for the sample size considerations). Recruitment bias will be evaluated by comparing key characteristics of the included participants, such as age, sex and BMI, with those of the BCSN.

The main inclusion and exclusion criteria for the BCSN trial and the CRCbiome study are listed in Table 1.

\section{Recruitment of participants}

Eligible subjects are invited after being informed about their positive FIT test and a colonoscopy appointment has been scheduled. Invitations to the CRCbiome study, including the two questionnaires, are sent out by mail a minimum of four days prior to the colonoscopy. Returning at least one of the two questionnaires is regarded as a consent to the study, and includes permission to collect, analyze and store fecal samples, and to retrieve information from questionnaires and health registries.

Both the BCSN trial and the CRCbiome study have been approved by the Regional Committee for Medical Research Ethics in South East Norway (Approval no.: $2011 / 1272$ and 63,148 , respectively). The BCSN is also registered at clinicaltrials.gov (Clinical Trial (NCT) no.: 01538550).

\section{Outcome variables}

For the first two aims, the outcome variable will be defined based on the colonoscopy result. Participants will be grouped into four main categories: no confirmed 
Table 1 Inclusion and exclusion criteria in the BCSN trial and CRCbiome study

\begin{tabular}{|c|c|}
\hline \multicolumn{2}{|c|}{ Inclusion criteria } \\
\hline \multirow[t]{2}{*}{$B C S N$} & Aged 50-74 years old in 2012 \\
\hline & $\begin{array}{l}\text { Resident in selected municipalities in South-East Norway } \\
\text { (Østfold, parts of Akershus and parts of Buskerud) }\end{array}$ \\
\hline CRCbiome & $\begin{array}{l}\text { FIT positive test (i.e. hemoglobin }>15 \mathrm{mcg} / \mathrm{g} \text { feces) and } \\
\text { invited to a follow-up colonoscopy }\end{array}$ \\
\hline \multicolumn{2}{|c|}{ Exclusion criteria } \\
\hline \multirow[t]{5}{*}{$B C S N^{2}$} & Death \\
\hline & Moving out of the area \\
\hline & Reaching the upper age limit \\
\hline & Diagnosed with CRC \\
\hline & Not attending screening colonoscopy \\
\hline \multirow{3}{*}{ CRCbiome } & \\
\hline & Low DNA concentration \\
\hline & Low sequencing yield (<2 gigabases) \\
\hline
\end{tabular}

${ }^{a}$ Exclusion criteria apply for individuals who died, moved out of the area, reached the upper age limit, or were diagnosed with CRC before they were due for invitation

neoplastic findings (Group 1); non-advanced lesions (Group 2); advanced lesions (Group 3); and CRC (Group 4) (Table 2). The advanced lesions group consists of both advanced adenomas (any adenoma with villous histology, high-grade dysplasia or polyp size greater than or equal to $10 \mathrm{~mm}$ ) and advanced serrated lesions (any serrated lesion with size $\geq 10 \mathrm{~mm}$ or dysplasia). In addition to separating by stage of the carcinogenic process, we may further subdivide lesions by clinicopathological features, including histopathological subtype (e.g. adenomas versus serrated lesions) and site of occurrence (proximal versus distal colon). Also of interest is the potential for distinct roles of environmental factors and the gut microbiome in the two main pathways of colorectal carcinogenesis: the adenoma-carcinoma pathway, and the serrated carcinoma pathway.

For the third aim, the outcome variable will be defined based on the metagenome data. We will monitor several aspects of the gut microbiome to describe the presence of bacterial strains and the functional potential in paired samples during re-establishment of the gut microbiome following bowel cleansing and colonoscopy.

Long-term effects in the study will be assessed 10 years after recruitment is completed. This will include an investigation of incidence and mortality of advanced colorectal lesions.

\section{Clinical data, biological sampling and questionnaires Assessment of clinical data}

As part of the BCSN [46], participants are contacted by a study nurse prior to follow-up colonoscopy, to obtain information on medical history. This includes prior
Table 2 Main outcomes of the screening colonoscopy among CRCbiome participants with preliminary distribution in percentages as of November 2020

\begin{tabular}{|c|c|}
\hline Colonoscopy result & Percentages $^{a}$ \\
\hline $\mathrm{FIT}+$, no colonoscopy & 3.6 \\
\hline \multicolumn{2}{|l|}{ Group 1} \\
\hline Negative & 11.2 \\
\hline Polyp without histology ${ }^{b}$ & 2.4 \\
\hline Non neoplastic findings & 18.2 \\
\hline \multicolumn{2}{|l|}{ Group 2} \\
\hline Non-advanced serrated lesions ${ }^{c}$ & 6.4 \\
\hline Non-advanced adenomas $(<3)$ & 23.6 \\
\hline Non-advanced adenomas $(\geq 3)$ & 8.4 \\
\hline \multicolumn{2}{|l|}{ Group 3} \\
\hline Advanced serrated lesions ${ }^{d}$ & 4.4 \\
\hline Advanced adenoma ${ }^{e}$ & 18.1 \\
\hline \multicolumn{2}{|l|}{ Group 4} \\
\hline $\mathrm{CRC}^{f}$ & 3.6 \\
\hline
\end{tabular}

${ }^{a}$ An extended version of this table, with colonoscopy result by FIT round, is shown in Additional file 1 (Supplementary Table 2). In cases of multiple findings, participants are allocated to the most severe group. Numbers will therefore add up to $100 \%$

${ }^{b}$ Polyps lost during colonoscopy or where the endoscopist considers biopsy unnescessary, for example hyperplastic polyps in the rectum

Includes hyperplastic polyps with size $<10 \mathrm{~mm}$ and sessile serrated lesions without dysplasia and size $<10 \mathrm{~mm}$

${ }^{d}$ Defined as any serrated lesions with size $\geq 10 \mathrm{~mm}$ or dysplasia

${ }^{e}$ Defined as any adenoma with either villous histology ( $225 \%$ villous

components), high-grade dysplasia or polyp size greater than or equal to $10 \mathrm{~mm}$ [52]

${ }^{f}$ Defined as presence of adenocarcinoma arising from the colon or rectum. Collectively, advanced adenoma or CRC are referred to as advanced neoplasia [52]

colonoscopies and CT colonographies, comorbidities, drug use, gastrointestinal symptoms, smoking habits, and body weight and height (Table 3 ). A variety of data are collected in relation to the follow-up colonoscopy, including screening outcomes (i.e. presence and clinicopathological characterization of detected lesions) and characteristics relevant to the endoscopic procedure (Table 3). For all lesions detected; size, location, appearance, technique used for removal and tissue sampling, and completeness of removal, are recorded. Both the medical history data and data collected as part of the follow-up colonoscopy, are entered into a dedicated database by the responsible health care provider. A complete overview of the data collected in the BCSN trial can be found elsewhere [46].

\section{Biological sampling and gut microbiome analysis}

FIT sampling and storage Sampling kits for stool sample collection are mailed to the participants three times during the study period, with the first sample being the positive BCSN FIT sample. No restrictions on diet or 


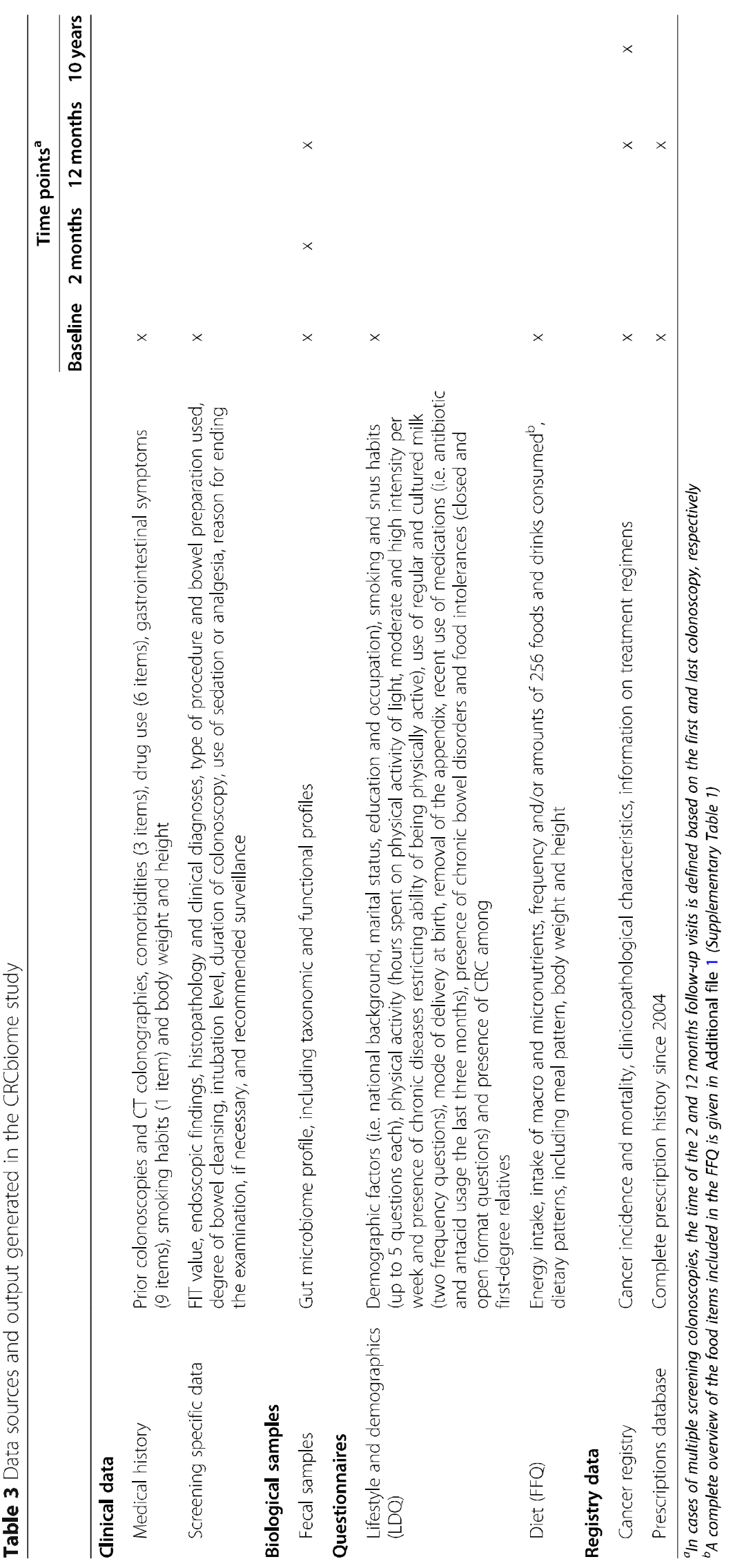


medication use are required prior to sampling. Stool is collected using plastic sticks, which collect about $10 \mathrm{mg}$ stool. The stool is then stored in $2 \mathrm{ml}$ of buffer containing HEPES (4-(2-hydroxyethyl)-1-piperazineethanesulfonic acid), BSA (Bovine serum albumin) and sodium azide. Samples are then packed in padded envelopes and returned by mail to a laboratory at Oslo University Hospital for analysis and further storage at $-80^{\circ} \mathrm{C}$. Shipping time is estimated to $3-10$ days. Immunochemical testing for blood in feces is performed continuously using the OC-Sensor Diana (Eiken Chemical, Tokyo, Japan) as samples are received at the laboratory.

DNA extraction We have shown that fecal matter collected in the FIT sampling procedure yields comparable microbial diversity and composition to fresh frozen stool samples [53].

Thawed samples are transferred to three $500 \mathrm{ml}$ aliquots from the sampling bottle using a blood sampling needle (Vacuette) perforating the plastic lid. Samples are stored at $-80^{\circ} \mathrm{C}$ until further processing.

Extraction of DNA is carried out using the QIAsymphony automated extraction system, using the QIAsymphony DSP Virus/Pathogen Midikit (Qiagen), after an offboard lysis protocol with some modifications. Each sample is lysed with bead-beating: a $500 \mu \mathrm{l}$ sample aliquot is transferred to a Lysing Matrix E tube (MP Biomedicals) and mixed with $700 \mu \mathrm{l}$ phosphate-buffered saline (PBS) buffer. The mixture is then shaken at $6.5 \mathrm{~m} / \mathrm{s}$ for $45 \mathrm{~s}$. After the bead-beating, $800 \mu \mathrm{l}$ of the sample is mixed with $1055 \mu \mathrm{l}$ of off-board lysis buffer (proteinase K, ATL buffer, ACL buffer and nuclease-free water) as recommended by Qiagen. The sample is incubated at $68^{\circ} \mathrm{C}$ for $15 \mathrm{~min}$ for lysis. Nucleic acid purification is performed on the QIAsymphony extraction robot using the Complex800_OBL_CR22796_ID 3489 protocol, a modified version of the Complex800_OBL_V4_DSP protocol. Purified DNA is eluted in $60 \mu \mathrm{l}$ AVE-buffer (Qiagen). DNA purity is assessed using a Nanodrop2000 (Thermo Fisher Scientific, USA), and the concentration is measured by Qubit (Thermo Fisher Scientific, USA).

Metagenome sequencing Libraries for metagenome sequencing are prepared from extracted DNA at the sequencing laboratory of the Institute for Molecular Medicine Finland FIMM Technology Centre, University of Helsinki (P.O. Box 20, University of Helsinki, Finland) using Illumina sequencing, with the aim of producing 3 gigabases of DNA sequence per sample.

In details, $29 \mu \mathrm{l}$ of extracted DNA is purified and concentrated by adding an equal volume of AMPure XP (Beckman Coulter Life Sciences, Indianapolis, IN, USA). Purification is then performed as per the manufacturer's instructions. The purified samples are eluted to $17 \mu \mathrm{l}$ of
$10 \mathrm{mM}$ Tris- $\mathrm{HCl}, \mathrm{pH} 8.5$, and DNA concentrations are determined by Quant-iT PicoGreen dsDNA Assay Kit (Thermo Fisher Scientific, Waltham, MA, USA). The samples are normalized to a maximum concentration of $3.3 \mathrm{ng} / \mu \mathrm{l}$, resulting in DNA inputs of $25 \mathrm{ng}$ or less.

Sequencing libraries are prepared according to the Nextera DNA Flex Library Prep Reference Guide (v07) (Illumina, San Diego, CA, USA), with the exception that the reaction volumes are scaled down to $1 / 4$ of the protocol volumes. The libraries are amplified according to the protocol with 7 PCR cycles. All the library preparation steps are performed on a Microlab STARlet (Hamilton Company, Reno, NV, USA) and Biomek NX ${ }^{\mathrm{P}}$ (Beckman Coulter Life Sciences, Indianapolis, IN, USA) liquid handlers running custom scripts.

DNA concentrations of the finished libraries are determined with Quant-iT PicoGreen dsDNA Assay. Libraries are combined into pools containing 240 libraries with $4.5 \mathrm{ng}$ of each library using Echo 525 Acoustic Liquid Handler (Beckman Coulter Life Sciences, Indianapolis, IN, USA). Library pools are size-selected to a fragment size range between 650 and $900 \mathrm{bp}$ using BluePippin (Sage Science Beverly, MA, USA).

Sequencing is performed with the Illumina NovaSeq system using S4 flow cells with lane divider (Illumina, San Diego, CA, USA). Each pool is sequenced on a single lane. Read length for the paired-end run is $2 \times 151 \mathrm{bp}$.

Processing and analysis of sequencing data Sequencing data are transferred to a platform for secure storage and analysis of sensitive research-related data at the University of Oslo [54]. The analysis of metagenomic sequencing data is handled in a uniform manner using a customizable workflow manager [55]. To establish a quality-filtered dataset, standard filters are applied: sequences corresponding to adapters used in library preparation, being of low quality [56] and those mapping to the human genome [57], with subsequent quality control of filtered sequencing reads [58].

Taxonomic classification and determination of microbial gene content, including functional annotation (e.g. using gene ontology and KEGG databases) will be performed using publicly available tools. Abundance measures will be used to calculate taxonomic and functional alpha and beta diversity, as well as serving as input for machine learning approaches aimed at producing classifiers for high-risk individuals in a data-driven manner. Further metagenome-derived measures may include identification of metagenome-assembled genomes, strain-level analysis and description of the gut virome.

\section{Questionnaires}

Two questionnaires are used to collect data on diet, lifestyle and demographic information; a food frequency 
questionnaire (FFQ) and a general lifestyle and demographics questionnaire (LDQ). Self-reported dates of questionnaire completion are registered in the project database. Returned questionnaires are reviewed manually before scanning and further processing. In cases of lowquality data, participants are contacted for clarification.

Assessment of dietary intake Dietary intake is assessed using a semiquantitative, 14-page FFQ, designed to assess the habitual diet during the preceding year. The questionnaire is a modified version of an FFQ developed and validated by the Department of Nutrition, University of Oslo [59-64]. The questionnaire has been validated for both energy intake [59-61], intake of macro and micronutrients $[59,61,64]$, as well as selected food items and groups [61-64]. The questionnaire includes 23 main questions, covering a total of 256 food items, as well as a free-text field for entries of food items not covered by the questionnaire. For each food item (except one on preferred types of fat for cooking), participants are asked to record frequency of consumption, ranging from never/seldom to several times a day, and/or amount, typically as portion size given in various household units (e.g. deciliters, glasses, cups, spoons). In total, there are 24.9 questions on frequency, 204 on portion size, one on preferences and nine other, mostly related to meal patterns (Additional file 1, supplementary Table 1).

As with any dietary assessment method, the FFQ is prone to errors due to inaccurate reporting and missing answers. Therefore, to mitigate such errors, a standardized framework for how to review and evaluate FFQ quality has been developed. A detailed overview of the framework is given in Additinoal file 2, supplementary Fig. 1. In brief, incoming FFQs are reviewed by trained personnel according to a set of predefined criteria. Scanning of questionnaires is performed using the Cardiff TeleForm program (Datascan, Oslo, Norway). The dietary calculation system KBS (short for "Kostberegningssystem"), developed at the Department of Nutrition, University of Oslo, is used to calculate food and nutrient intake. The latest version of the food database (i.e. AE18 or newer) will be used, which is largely based on the Norwegian Food Composition Table [65]. In line with common practice in nutrition studies, missing answers are imputed as zero intake $[61,63,66,67]$ and observations with extreme energy intake levels in both the upper and lower range will be excluded [68].

The main focus of the dietary analyses will be on foods and drinks linked to the risk of CRC and its precursor lesions, including intakes of alcohol, red and processed meat, wholegrains, foods containing dietary fiber, dairy products and calcium supplements [69]. Dietary intake will also be studied holistically by employing various dietary indices such as the 2018 World Cancer Research Fund/American Institute for Cancer Research (WCRF/ AICR) index for adherence to cancer prevention recommendations [70].

Assessment of lifestyle and demographic data Lifestyle and demographic data are assessed using a four page questionnaire based on questions used in previous national surveys $[71,72]$. Prior to the study start, the questionnaire was piloted in a targeted population and adjusted based on feedback from pilot study participants. The questionnaire has ten main questions covering demographic factors (national background, education, occupation and marital status), diagnosis of CRC among first-degree relatives, presence of chronic bowel disorders and food intolerances, removal of the appendix, mode of delivery at birth, smoking and snus (i.e. smokeless tobacco) habits, recent use of medications, the past years' physical activity level and use of regular and cultured milk, which is not completely covered in the FFQ (see Table 3 for a detailed overview). In the questions concerning smoking and snus habits, participants are asked to recall their current habits, including the daily number of cigarettes/snus portions, as well as years since possible cessation and total years of use. Questionnaires are scanned and processed using the Cardiff TeleForm program (InfoShare, Oslo, Norway).

\section{Registry data}

Data collected in the CRCbiome study will be linked to national registries, including the Norwegian Prescription Database and the Cancer Registry of Norway, using personal identification numbers. Complete data linkages will be undertaken twice during active follow-up: after all participants have completed baseline and diagnostic information from follow-up colonoscopies is available, and then after the one-year follow-up is completed. In addition, linkage to the Cancer Registry of Norway will be performed at least once during the 10 year follow-up period.

\section{Norwegian prescription database}

The Norwegian Prescription Database [73] will be used to obtain information on medication history prior to CRC screening, and during the first year of follow-up. The registry contains data on all medications prescribed to Norwegian citizens since 2004. Prescription drugs are categorized according to the Anatomical Therapeutic Chemical (ATC) system, a hierarchical classification system developed by the WHO [74, 75]. For each drug, the number of packages dispensed, the number of defined daily doses (DDD), the prescription category, and the date of dispensing are registered. 
Linkage to the Norwegian Prescription Database enables an in-depth analysis of associations between drug use, the gut microbiome and advanced colorectal lesions. Initially, we will perform drug-wide association analyses to screen for potential associations, adjusting for key covariates. Detected associations will then be examined in detail, including a more refined categorization of drug variables, robust covariate adjustments as well as the analysis of timing and dose-response relations. Prescription histories will also be used as a proxy for life-long burden of chronic diseases. To examine the representativeness of the drug profiles discoverd in the CRCbiome study, a randomly selected control group drawn from the National Population Registry, might be included.

\section{Cancer registry of Norway}

Information on clinicopathological characteristics, cancer therapy, as well as outcomes assessed as part of passive follow-up, will be obtained from the Cancer Registry of Norway [76]. The Cancer Registry of Norway has recorded incident cancer cases on a nationwide basis since 1953 and has been shown to have accurate and almost complete ascertainment of cases $(98.8 \%$ for the registration period 2001-2005) [77]. According to recent estimates, about $93 \%$ of all cancer cases and $\geq 95 \%$ of cancers in the colon and rectum are morphologically verified [48]. Cancer diagnoses are recorded using the International Classification of Diseases, version 10 (ICD10). Mortality data in the registry are obtained from the Cause of Death Registry and coded using the same ICD10 categories as for the incidence data.

\section{Data processing and management}

To facilitate project administration, including recruitment and follow-up of participants, custom software has been developed. This application communicates with two project specific databases (i.e. the BCSN and CRCbiome databases). Only authorized data manager personnel have complete access to the datasets. A simplified version of the data generation process is depicted in Fig. 2.

In line with common practice for linkage with national registries [78], linked data will receive unique ID numbers specific to the particular project. Linkage of research data will be performed by the data controller. For the metagenome data, which due to its size cannot be

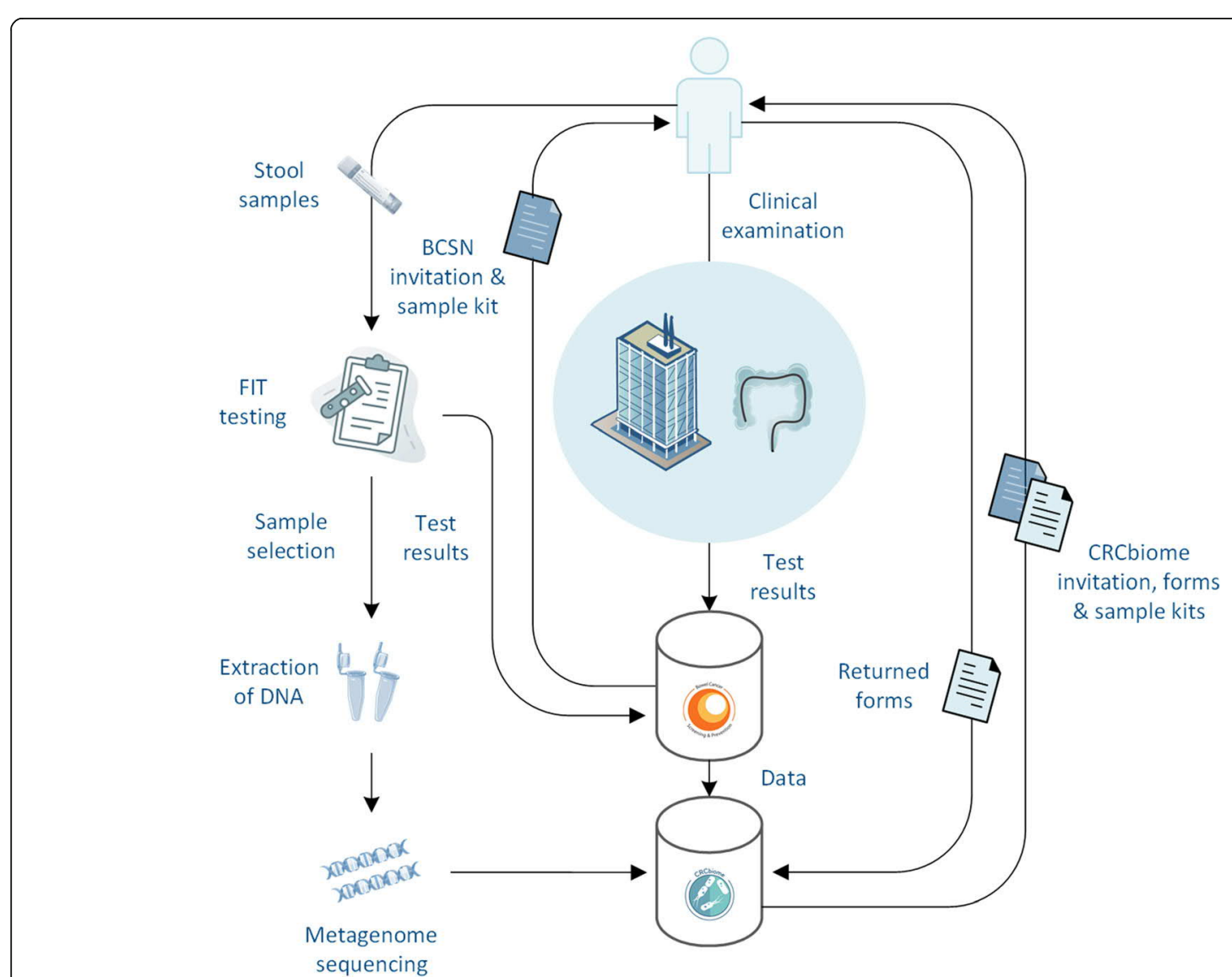

Fig. 2 Simplified version of the data generation process in CRCbiome. The figure is created based on free images from Servier Medical Art (Creative Commons Attribution Liscence, creativecommons.org/liscences/by/3.0/) and Stockio (https://www.stockio.com/) 
transferred using ordinary methods, linkage will be performed in-house by an independent data manager without access to other parts of the data than those strictly necessary for linkage.

All data collected in the CRCbiome study will be stored and analyzed at a platform for secure handling of sensitive research-related data, operated by the University of Oslo [54]. Access to research data for external investigators, or use outside of the current protocol, will require approval from the Norwegian Regional Committee for Medical and Health Research Ethics and a data access committee (information available on the project web site [79]). Research data are not openly available because of the principles and conditions set out in articles 6 [1] (e) and 9 [2] (j) of the General Data Protection Regulation (GDPR).

\section{Statistical analyses and sample size considerations}

The number of participants to include was chosen with the aim of providing adequate power for the development of a highly sensitive classification algorithm via data-driven analyses of gut metagenomes that will accurately identify FIT-positive individuals in need of clinical intervention.

The classifier will be trained using counts of taxonomic units, signature and genes categorized according to gene ontology or pathway membership from metagenomes, FFQ, demographic and lifestyle data as input variables, and advanced colorectal lesions as outcome (i.e. group group 3 and group 4, Table 2). The CRC risk classification will be done using machine learning algorithms suited to metagenome data, such as lasso regression [80], support-vector machines [81], random forests [82], multi-layer perception neural networks [83] and scalable tree boosting [84] algorithms. Evaluation of the classifier will be conducted in a leave-out test set. As outlined below, we believe that with sufficient sample size, development of a classifier with a sensitivity of 0.95 is achievable in the training set, being within the range of published reports [30, 33].

Interpretation of the classifier will be sought by post hoc analysis, quantifing the importance of individual features (taxa, genes and pathways) in making predictions. Stratified analyses will be done to evaluate the classifier within different subgroups of the population (e.g. by age group, sex and screening center).

With a projected classifier sensitivity of 0.95 and a minimally acceptable sensitivity of 0.8 , at $80 \%$ power and 95\% confidence level, 50 participants with advanced colorectal lesions are required in the test set [85]. Classifier specificity in the setting of FIT-positive individuals will have a lower requirement, and we therefore set the expected classifier specificity to 0.75 and a minimally acceptable specificity of 0.6 , thus requiring 100 participants with normal findings in the test set. Based on initial recruitment, we expect a participation rate of $58 \%$, with $26 \%$ of participants having findings of advanced lesions or CRC (Table 2). By inviting 2700 FIT-positive BCSN participants, and splitting the training and test sets 80 / 20, a projected number of 1253 and 313 participants will constitute the training and test sets, respectively, which will include adequate numbers of participants with both advanced colorectal lesions and normal findings in the test set. With this sample size, we will also be able to perform stratified analyses. The machine learning analyses will be complemented by various multivariate regression analyses, stratified by the covariates outlined above.

\section{Discussion}

CRC remains a major public health challenge with substantial personal and societal costs [22]. Screening is an effective measure to reduce disease burden [22]. However, current screening methods suffer from limitations, limiting the number of preventable cases. Innovative use of currently available methods represents a promising avenue for improvements in CRC prevention [22]. The current study is designed to contribute to the development of microbial biomarkers, using metagenome sequencing and comprehensive questionnaire and registry data for improved detection of advanced lesions and CRC in a FIT-positive population. The CRCbiome study is unique in that it uses data from the screening population to develop relevant biomarkers.

The idea of using microbial biomarkers to increase the performance of CRC screening has received increased attention with the adoption of high-throughput characterization of the gut microbiome. Ideally, combining microbial biomarkers with FIT testing could achieve the sensitivity of direct visualization methods and the uptake of non-invasive fecal tests. Several studies have demonstrated improved ability to discriminate individuals with healthy colons from those with advanced neoplasia when adding microbial biomarkers in the prediction model, more so for carcinoma (area under the curve (AUC) of 0.87-0.97 [30, 33, 34]) than adenoma (AUC of 0.76 [33]). Despite great promise, these studies have typically been limited by small sample sizes [30, 32-34], cross-sectional designs [30-34], use of suboptimal or low-resolution methods to study the gutmicrobiome [30-33] and lack of data on important confounders [30-34]. The CRCbiome study seeks to address several of these shortcomings.

Major strengths of the CRCbiome study include its large sample size and prospective nature, use of state of the art methodology for studying the gut microbiome and access to detailed information on likely confounders of the relationship between the gut-microbiome and 
advanced colorectal lesions. A further strength of the study is in its organization and logistics structure, being nested within the BCSN. The immediate availability of clinically verified outcome data, via follow-up colonoscopies and cancer registry data, allow for prospective investigations on multiple outcomes relevant to the screening population (e.g. polyp recurrence). Access to comprehensive high-quality data on diet and lifestyle, including complete prescription histories, also enables the investigation of the predictive performance of more broad classifiers, laying the ground for personalized screening strategies, including risk-stratified approaches.

With a study population solely consisting of FIT positive participants, the projected number of individuals with high-risk lesions or CRC is relatively high (about 409 (26\%), group 3 and 4 , Table 2), thereby increasing the power to achieve accurate classification of advanced colorectal neoplasms. Still, whether findings in this population extends to cases missed by FIT testing is unknown.

Collection of follow-up samples at 2 and 12-months post colonoscopy represents an extension of the crosssectional design of most prior studies, shedding light on the development of the gut microbiome following colonoscopy with or without removal of CRC precursor lesions. While there are examples of shifts in microbial profiles following colonoscopy, the gut microbiome typically reverts to the initial state within weeks [86]. Deviations from re-establishment of the gut microbiome both in the medium and long term have the potential for causal interpretations.

The study also has some limitations. Exclusive selection of FIT positive participants may limit the generalizability of the findings to those with bleeding neoplastic lesions. Consequently, improvements in diagnostic performance may be limited to specificity, and thus the ability to correctly classify healthy individuals. However, since lesions tend to bleed intermittently [87] and the study aims to identify potential causal pathways, we consider it likely that the identified biomarker also may have improved sensitivity in the screening population as a whole.

A further limitation is the lack of information on fecal metrics such as the Bristol stool scale, which has been shown to be an important determinant of microbiota richness and variance [88]. However, variation in microbiome profile due to stool consistency could likely be explored by use of gastrointestinal symptoms as a surrogate, data on which is available in the BCSN database.

Lastly, lack of follow-up data on diet and lifestyle may complicate the interpretation of microbial changes following colonoscopy. Even though prior studies in comparable study populations show that potential changes in diet and lifestyle following screening are modest $[89,90]$, caution in interpretation of follow-up samples is warranted.
The CRCbiome study represents a valuable source of data for further research. An example is access to complete prescription histories from the Norwegian Prescription Database that enables in-depth analyses of associations between a broad range of medications, microbial features and neoplasia risk, both during short and long-term follow-up. The fecal samples collected are also biobanked and can be used for other purposes beside the study aims of the current protocol. For instance, in addition to metagenome sequencing, the fecal samples can potentially be used for other omics analyses, such as transcriptome and metabolome analysis. All tissue specimens removed during colonoscopy are also available to the project, enabling in-depth molecular profiling.

The integration of a microbiome-based biomarker into national CRC screening programs is a long-term process, requiring many steps before enabling full implemtation. Ideally, the discovery phase will lead to the identification of a few selected features that will predict the occcurence of advanced colorectal lesions with high accuracy. These could then be combined by means of a biomarker panel for the development of a rapid test, which, following rigorous validation and testing, has the potential of being integrated into screening programs. The cost-effectivness of adding a microbial biomarker to the FIT test should be carefully evaluated before implementation.

\section{Conclusion}

The CRCbiome study investigates the role of the gut microbiome, and its interactions with host factors, diet and lifestyle, in early stage colorectal carcinogenesis. Information obtained from this project will guide the development of a microbial biomarker for accurate detection of advanced colorectal lesions. By performing biomarker discovery within a screening population, the generalizability of the findings to future screening cohorts is likely to be high.

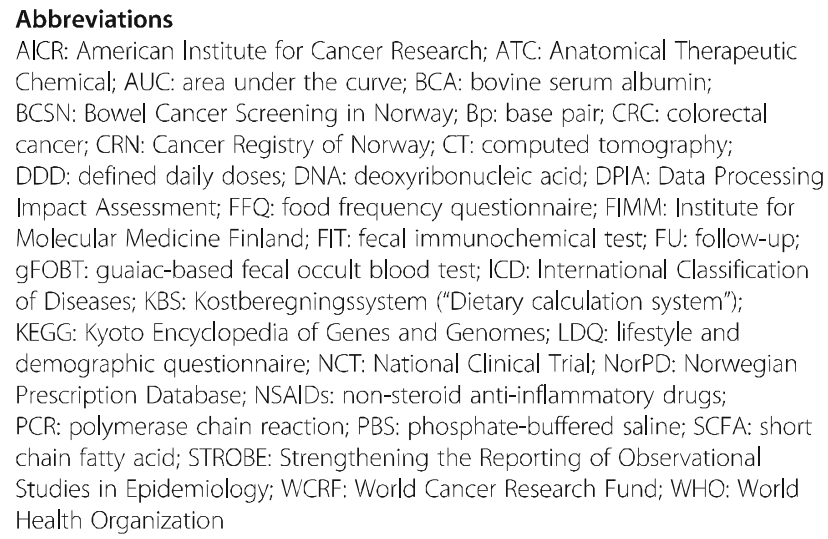

\section{Supplementary Information}

The online version contains supplementary material available at https://doi. org/10.1186/s12885-021-08640-8. 
Additional file 1. Contains two supplementary tables.

Additional file 2. Contains a supplementary figure with figure title and legend.

Additional file 3. Contains the STROBE checklist for observational studies.

\section{Acknowledgements}

We would like to aknowledge the devoted secretaries, nurses and doctors at Bærum and Moss hospital, and the biomedical laboratory scientists at Oslo University Hospital for their contributions to this study. We would also like to thank the personnel involved in sequencing all CRCbiome samples at the Sequencing laboratory of Institute for Molecular Medicine Finland FIMM Technology Centre, University of Helsinki. Lastly, we would like to thank each study participoant, as well as all collaborative partners, technicians and students that have, and will, contribute to this study.

\section{Authors' contributions}

ASK and EB1 had the main responsibility for writing the manuscript. ASK EB1, CBJ, EV, JIN, HK, VB, PE, EB2, EN, TR, EH, RL1, OHA, WMdV, SB, AH, RL2, $M S, H S B, G U, K R R, T d L, G H, \varnothing H, P B$ and TBR contributed to the study design and protocol. ASK, EB1, CBJ, EV, JIN, HK, VB, PE, EB2, EN, TR, EH, RL1, OHA, WMdV, SB, AH, RL2, MS, HSB, GU, KRR, TdL, GH, ØH, PB and TBR contributed to the writing and approval of the final manuscript. $P B$ and TBR are the principal investigators.

\section{Funding}

This project would not have been possible without funding from the Norwegian Cancer Society, the Research Council of Norway and the South Eastern Norway Regional Health Authority. The funding bodies played no role in the design of the study and collection, analysis, and interpretation of data and in writing the manuscript.

\section{Availability of data and materials}

Due to the principles and conditions set out in articles 6 [1] (e) and 9 [2] (j) of the General Data Protection Regulation (GDPR), research data generated in the CRCbiome study are not openly available. Further information on access to CRCbiome data can be found on the project web site [79]).

\section{Declarations}

Ethics approval and consent to participate

The CRCbiome study is approved by the Norwegian Regional Committees for Medical and Health Research Ethics (Approval no.: 63148). Returning at least one of the two questionnaires sent out to potential study participants at baseline is regarded as a consent to the study, and includes permission to collect, analyze and store fecal samples, and to retrieve information from questionnaires and health registries. All biological materials are stored in a biobank at Oslo University Hospital.

\section{Consent for publication}

Not applicable.

\section{Competing interests}

There are no competing interests.

\section{Author details}

Section for Colorectal Cancer Screening, Cancer Registry of Norway, Oslo, Norway. ${ }^{2}$ Department of Research, Cancer Registry of Norway, Oslo, Norway. ${ }^{3}$ Department of Tumor Biology, Institute for Cancer Research, The Norwegian Radium Hospital, Oslo University Hospital, Oslo, Norway. ${ }^{4}$ Department of Medical Biochemistry, Oslo University Hospital, Oslo, Norway. 'Institute for Molecular Medicine Finland, HiLIFE, University of Helsinki, Helsinki, Finland. ${ }^{6}$ Department of Multidisciplinary Laboratory Science and Medical Biochemistry, Genetic Unit, Akershus University Hospital, Lørenskog, Norway. ${ }^{7}$ Department of Informatics, Centre for Bioinformatics, University of Oslo, Oslo, Norway. ${ }^{8}$ Department of Microbiology, Oslo University Hospital, Oslo, Norway. ${ }^{9}$ Department of Medical Genetics, Oslo University Hospital and University of Oslo, Oslo, Norway. ${ }^{10} \mathrm{Centre}$ for Fertility and Health, Norwegian Institute of Public Health, Oslo, Norway. ${ }^{11}$ Department of Microbiology and
Infection Control, Akershus University Hospital, Lørenskog, Norway. ${ }^{12}$ Department of Natural Sciences and Health, Oslo Metropolitan University, Oslo, Norway. ${ }^{13}$ Laboratory of Microbiology, Wageningen University, Wageningen, The Netherlands. ${ }^{14}$ Human Microbiome Research Program, Faculty of Medicine, University of Helsinki, Helsinki, Finland. ${ }^{15}$ Department of Genetics and Lineberger Comprehensive Cancer Center, University of North Carolina, Chapel Hill, NC, USA. ${ }^{16}$ Department of Nutrition, University of Oslo, Oslo, Norway. ${ }^{17}$ Department of Biology and Biological Engineering, Division of Food and Nutrition Science, Chalmers University of Technology, Gothenburg, Sweden. ${ }^{18}$ Department of Epidemiology, Harvard T.H. Chan School of Public Health, Boston, MA, USA. ${ }^{19}$ Department of Nutrition, Harvard T.H. Chan School of Public Health, Boston, MA, USA. ${ }^{20} \mathrm{Clinical}$ and Translational Epidemiology Unit and Division of Gastroenterology, Massachusetts General Hospital and Harvard Medical School, Boston, MA, USA. ${ }^{21}$ Department of Drug Statistics, Norwegian Institute of Public Health, Oslo, Norway. ${ }^{22}$ School of Pharmacy, University of Oslo, Oslo, Norway. ${ }^{23}$ Cancer Registry of Norway, Oslo, Norway. ${ }^{24}$ Medical Department, Sahlgrenska University Hospital-Mölndal, Mölndal, Sweden. ${ }^{25}$ Department of Molecular and Clinical Medicine, Sahlgrenska Academy, University of Gothenburg, Gothenburg, Sweden. ${ }^{26}$ Department of Medical Research, Bærum Hospital, Bærum, Norway. ${ }^{27}$ Department of Research, Telemark Hospital, Skien, Norway. ${ }^{28}$ Department of Medicine, Sorlandet Hospital Kristiansand, Kristiansand, Norway. ${ }^{29}$ Institute for Health and Society, University of Oslo, Oslo, Norway.

Received: 16 December 2020 Accepted: 27 July 2021 Published online: 18 August 2021

\section{References}

1. Bray F, Ferlay J, Soerjomataram I, Siegel RL, Torre LA, Jemal A. Global cancer statistics 2018: GLOBOCAN estimates of incidence and mortality worldwide for 36 cancers in 185 countries. CA Cancer J Clin. 2018;68(6):394-424.

2. Ouakrim DA, Pizot C, Boniol M, Malvezzi M, Boniol M, Negri E, et al. Trends in colorectal cancer mortality in Europe: retrospective analysis of the $\mathrm{WHO}$ mortality database. BMJ. 2015:351:1-10.

3. Safiri S, Sepanlou SG, Ikuta KS, Bisignano C, Salimzadeh H, Delavari A, et al. The global, regional, and national burden of colorectal cancer and its attributable risk factors in 195 countries and territories, 1990-2017: a systematic analysis for the global burden of disease study 2017. Lancet Gastroenterol Hepatol. 2019;4(12):913-33. https://doi.org/10.1016/52468-12 53(19)30345-0

4. Danckert B, Ferlay J, Engholm G, Hansen HL, Johannesen TB, Khan S, et al NORDCAN: Cancer Incidence, Mortality, Prevalence and Survival in the Nordic Countries. Version 8.2. 2019.

5. Howlader N, Noone AM, Krapcho M, Miller D, Brest A, Yu M, et al (eds). SEER Cancer Statistics Review, 1975-2017, National Cancer Institute., Bethesda, MD, https://seer.cancer.gov/csr/1975_2017/, based on November 2019 SEER data submission, posted to the SEER web site, April 2020. 2020.

6. Sung $H$, Siegel RL, Rosenberg PS, Jemal A. Emerging cancer trends among young adults in the USA: analysis of a population-based cancer registry. Lancet Public Heal. 2019;4:e137-47.

7. Araghi M, Soerjomataram I, Bardot A, Ferlay J, Cabasag CJ, Morrison DS, et al. Changes in colorectal cancer incidence in seven high-income countries: a population-based study. Lancet Gastroenterol Hepatol. 2019; 4(7):511-8. https://doi.org/10.1016/S2468-1253(19)30147-5.

8. Leggett $B$, Whitehall V. Role of the serrated pathway in colorectal Cancer pathogenesis. Gastroenterology. 2010;138(6):2088-100. https://doi.org/10.1 053/j.gastro,2009.12.066.

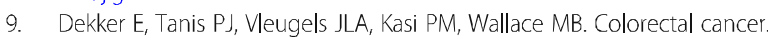
Lancet (London, England). 2019;394:1467-80 http://www.ncbi.nlm.nih.gov/ pubmed/31631858.

10. Bibbins-Domingo K, Grossman DC, Curry SJ, Davidson KW, Epling JW, García FAR, et al. Screening for colorectal cancer: US preventive services task force recommendation statement. JAMA - J Am Med Assoc. 2016;315:2564-75.

11. Segnan N, Patnick J. von Karsa L EC. European guidelines for quality Assurance in Colorectal Cancer Screening and Diagnosis - first edition. Luxembourg: Office for Official Publications of the European Communities; 2010.

12. Ebell MH, Thai TN, Royalty KJ. Cancer screening recommendations: an international comparison of high income countries. Public Health Rev. 2018; 39:1-19. 
13. Sung JJY, Ng SC, Chan FKL, Chiu HM, Kim HS, Matsuda T, et al. An updated Asia Pacific consensus recommendations on colorectal cancer screening. Gut. 2015;64(1):121-32. https://doi.org/10.1136/gutjnl-2013-306503.

14. Atkin W, Wooldrage K, Parkin DM, Kralj-Hans I, MacRae E, Shah U, et al. Long term effects of once-only flexible sigmoidoscopy screening after 17 years of follow-up: the UK Flexible Sigmoidoscopy Screening randomised controlled trial. Lancet. 2017;389:1299-1311. https://doi.org/10.1016/50140-673 6(17)30396-3 Accessed 8 June 2021.

15. Schoen R, Pinsky P, Weissfeld J, Yokochi L, Church T, Laiyemo A, et al Colorectal-Cancer incidence and mortality with screening flexible sigmoidoscopy. N Engl J Med. 2012;366(25):2345-57. https://doi.org/10.1 056/NEJMoa1114635.

16. Segnan N, Armaroli P, Bonelli L, Risio M, Sciallero S, Zappa M, et al. Onceonly sigmoidoscopy in colorectal cancer screening: follow-up findings of the italian randomized controlled trial - SCORE. J Natl Cancer Inst. 2011; 103(17):1310-22. https://doi.org/10.1093/jnci/djr284

17. Holme $\varnothing$, Løberg $M$, Kalager $M$, Bretthauer $M$, Hernán MA, Aas E, et al. Effect of flexible sigmoidoscopy screening on colorectal cancer incidence and mortality: a randomized clinical trial. JAMA - J Am Med Assoc. 2014;312(6): 606-15. https://doi.org/10.1001/iama.2014.8266.

18. Mandel J, Bond J, Church T, Snover D, Bradley M, Schuman L, et al. Reducing mortality from colorectal cancer by screening for fecal occult blood. Minnesota Colon Cancer Control Study. N Engl J Med. 1993;

19. Kronborg O, Fenger C, Olsen J, Jørgensen OD, Søndergaard O. Randomised study of screening for colorectal cancer with faecal-occult-blood test. Lancet. 1996;348(9040):1467-71. https://doi.org/10.1016/S0140-6736(96)03430-7.

20. Lindholm E, Brevinge $H$, Haglind $E$. Survival benefit in a randomized clinical trial of faecal occult blood screening for colorectal cancer. Br I Surg England. 2008;95(8):1029-36. https://doi.org/10.1002/bjs.6136.

21. Hardcastle JD, Chamberlain JO, Robinson MHE, Moss SM, Amar SS, Balfour TW, et al. Randomised controlled trial of faecal-occult-blood screening for colorectal cancer. Lancet. 1996;348(9040):1472-7. https://doi.org/10.1016/ 50140-6736(96)03386-7.

22. Ladabaum U, Dominitz JA, Kahi C, Schoen RE. Strategies for Colorectal Cancer Screening. Gastroenterology [Internet]. Elsevier, Inc; 2020;158:418. https://doi.org/10.1053/.gastro.2019.06.043.

23. Schreuders EH, Ruco A, Rabeneck L, Schoen RE, Sung JJY, Young GP, et al. Colorectal cancer screening: a global overview of existing programmes. Gut 2015;64(10):1637-49. https://doi.org/10.1136/gutjnl-2014-309086.

24. Haug U, Kuntz KM, Knudsen AB, Hundt S, Brenner H. Sensitivity of immunochemical faecal occult blood testing for detecting left-vs rightsided colorectal neoplasia. Br J Cancer. 2011;104(11):1779-85, https://doi. org/10.1038/bjc.2011.160.

25. Holme $\varnothing$, Løberg $M$, Kalager $M$, Bretthauer $M$, Hernán MA, Aas $E$, et al. Long-term effectiveness of sigmoidoscopy screening on colorectal cancer incidence and mortality in women and men: a randomized trial. Ann Intern Med. 2018:168(11):775-82. https://doi.org/10.7326/M17-1441.

26. Brenner $H$, Qian J, Werner $S$. Variation of diagnostic performance of fecal immunochemical testing for hemoglobin by sex and age: results from a large screening cohort. Clin Epidemiol. 2018;10:381-9. https://doi.org/10.214 7/CLEP.S155548

27. Tilg H, Adolph TE, Gerner RR, Moschen AR. The Intestinal Microbiota in Colorectal Cancer. Cancer Cell. Elsevier Inc:; 2018;33:954-964.

28. Kostic AD, Gevers D, Pedamallu CS, Michaud M, Duke F, Earl AM et al. Genomic analysis identifies association of Fusobacterium with colorectal carcinoma. Genome Res. 2012;22(2):292-8. https://doi.org/10.1101/gr.12 6573.111.

29. Castellarin M, Warren R, Freeman J, Dreolini L, Krzywinski M, Strauss J, et al. Fusobacterium nucleatum infection is prevalent in human colorectal carcinoma. Genome Res. 2012;22(2):299-306. http://www.embase.com/ search/results?subaction=viewrecord\&from=export\&id $=\mathrm{L} 364186952 \% 5 \mathrm{C}$ nhttp://genome.cshlp.org/content/22/2/299.full.pdf+html\%5Cnhttp://dx.doi, org/10.1101/gr.126516.111\%5Cnhttp://sfx.library.uu.nl/utrecht?sid= EMBASE\&issn $=10889051 \& \mathrm{id}=$ doi:10

30. Zackular JP, Rogers MAM, Ruffin MT, Schloss PD. The human gut microbiome as a screening tool for colorectal cancer. Cancer Prev Res. 2014 7(11):1112-21. https://doi.org/10.1158/1940-6207.CAPR-14-0129.

31. Guo $S$, Li L, Xu B, Li M, Zeng $Q$, Xiao H, et al. A simple and novel fecal biomarker for colorectal cancer: ratio of Fusobacterium nucleatum to probiotics populations, based on their antagonistic effect. Clin Chem. 2018; 64(9):1327-37. https://doi.org/10.1373/clinchem.2018.289728.
32. Liang Q, Chiu J, Chen Y, Huang Y, Higashimori A, Fang J, et al. Feca bacteria act as novel biomarkers for noninvasive diagnosis of colorectal cancer. Clin Cancer Res. 2017;23(8):2061-70. https://doi.org/10.1158/10780432.CCR-16-1599.

33. Baxter NT, Ruffin MT, Rogers MAM, Schloss PD. Microbiota-based model improves the sensitivity of fecal immunochemical test for detecting colonic lesions. Genome Med. 2016;8(1):1-10. https://doi.org/10.1186/s13 073-016-0290-3.

34. Zeller G, Tap J, Voigt AY, Sunagawa S, Kultima JR, Costea PI, et al. Potential of fecal microbiota for early-stage detection of colorectal cancer. Mol Syst Biol. 2014;10(11):766. https://doi.org/10.15252/msb.20145645.

35. Ahn J, Sinha R, Pei Z, Dominianni C, Wu J, Shi J, et al. Human gut microbiome and risk for colorectal cancer. J Natl Cancer Inst. 2013;105(24): 1907-11. https://doi.org/10.1093/jnci/djt300.

36. Vogtmann $E$, Hua X, Zeller G, Sunagawa S, Voigt AY, Hercog R, et al Colorectal cancer and the human gut microbiome: reproducibility with whole-genome shotgun sequencing. PLoS One. 2016;11:1-13.

37. Feng $\mathrm{Q}$, Liang $\mathrm{S}$, Jia $\mathrm{H}$, Stadlmayr $\mathrm{A}$, Tang $\mathrm{L}$, Lan $\mathrm{Z}$, et al. Gut microbiome development along the colorectal adenoma-carcinoma sequence. Nat Commun. 2015;6(1). https://doi.org/10.1038/ncomms7528.

38. Yu J, Feng Q, Wong SH, Zhang D, Yi Liang Q, Qin Y, et al. Metagenomic analysis of faecal microbiome as a tool towards targeted non-invasive biomarkers for colorectal cancer. Gut. 2017;66(1):70-8. https://doi.org/10.113 6/gutjnl-2015-309800

39. Hale VL, Chen J, Johnson S, Harrington SC, Yab TC, Smyrk TC, et al. Shifts in the fecal microbiota associated with adenomatous polyps. Cancer Epidemiol Biomark Prev. 2017;26(1):85-94. https://doi.org/10.1158/1055-9965.EPI-16-0337.

40. Rothschild D, Weissbrod O, Barkan E, Kurilshikov A, Korem T, Zeevi D, et al. Environment dominates over host genetics in shaping human gut microbiota. Nature Publishing Group; 2018;

41. Song $M$, Chan AT, Sun J. Influence of the gut microbiome, diet, and environment on risk of colorectal Cancer. Gastroenterology. 2020;158(2): 322-40. https://doi.org/10.1053/j.gastro.2019.06.048

42. Song M, Chan AT. Environmental Factors, Gut Microbiota, and Colorectal Cancer Prevention. Clinical Gastroenterology and Hepatology. Am Gastroenterological Association; 2019. 275-289 p. https://doi.org/10.1016/j. cgh.2018.07.012.

43. Scott AJ, Alexander J, Merrifield CA, Cunningham D, Jobin C, Brown R, et al. International Cancer microbiome consortium consensus statement on the role of the human microbiome in carcinogenesis. Gut. 2019;68(9):162432. https://doi.org/10.1136/gutinl-2019-318556.

44. Thomas AM, Manghi P, Asnicar F, Pasolli E, Armanini F, Zolfo M, et al. Metagenomic analysis of colorectal cancer datasets identifies cross-cohort microbial diagnostic signatures and a link with choline degradation. Nat Med. 2019;25(4):667-78. https://doi.org/10.1038/s41591-019-0405-7.

45. Wirbel J, Pyl PT, Kartal E, Zych K, Kashani A, Milanese A, et al. Meta-analysis of fecal metagenomes reveals global microbial signatures that are specific for colorectal cancer. Nat Med. 2019;25(4):679-89. https://doi.org/10.1038/ \$41591-019-0406-6.

46. Randel KR, Schult AL, Botteri E, Hoff G, Bretthauer M, Ursin G, et al. Colorectal cancer screening with repeated fecal immunochemical test versus sigmoidoscopy: baseline results from a randomized trial. United States: Gastroenterology; 2020

47. Norwegian Institute of Public Health (NIPH). Norwegian Prescription Database (NorPD). https://www. fhi.no/en/hn/health-registries/norpd/. Accessed 5 November 2020.

48. Cancer Registry of Norway. Cancer in Norway 2019 - Cancer incidence, mortality, survival and prevalence in Norway. Oslo; 2020

49. Vandenbroucke JP, Von Elm E, Altman DG, Gøtzsche PC, Mulrow CD, Pocock $\mathrm{SJ}$, et al. Strengthening the reporting of observational studies in epidemiology (STROBE): explanation and elaboration. PLoS Med. 2007:4:1628-54.

50. Vandenbroucke JP, von Elm E, Altman DG, Gøtzsche PC, Mulrow CD, Pocock $S J$, et al. Strengthening the reporting of observational studies in epidemiology (STROBE): explanation and elaboration. Int I Surg. 2014;12(12): 1500-24. https://doi.org/10.1016/j.ijsu.2014.07.014

51. Bharucha T, Oeser C, Balloux F, Brown JR, Carbo EC, Charlett A, et al. STROBE-metagenomics: a STROBE extension statement to guide the reporting of metagenomics studies. Lancet Infect Dis. 2020;3099(10):1-10. https://doi.org/10.1016/51473-3099(20)30199-7

52. Hassan C, Quintero E, Dumonceau JM, Regula J, Brandão C, Chaussade S, et al. Post-polypectomy colonoscopy surveillance: European Society of 
Gastrointestinal Endoscopy (ESGE) guideline. Endoscopy. 2013;45(10):842-51. https://doi.org/10.1055/s-0033-1344548.

53. Rounge TB, Meisal R, Nordby J, Ambur OH, De Lange T, Hoff G. Evaluating gut microbiota profiles from archived fecal samples. BMC Gastroenterol. 2018;18:1-11.

54. University of Oslo (UiO). About TSD. https://www.uio.no/english/services/it/ research/sensitive-data/about/index.html. Accessed 14 December 2020.

55. Köster J, Rahmann S. Snakemake - a scalable bioinformatics workflow engine. Bioinformatics. 2018;34(20):3600. https://doi.org/10.1093/bioinforma tics/bty350.

56. Bolger AM, Lohse M, Usadel B. Trimmomatic: a flexible trimmer for Illumina sequence data. Bioinformatics. 2014;30(15):2114-20. https://doi.org/10.1093/ bioinformatics/btu170.

57. Langmead B, Salzberg SL. Fast gapped-read alignment with bowtie 2. Nat Methods. 2012;9(4):357-9. https://doi.org/10.1038/nmeth.1923.

58. Ewels P, Magnusson M, Lundin S, Käller M. MultiQC: summarize analysis results for multiple tools and samples in a single report. Bioinformatics. 2016;32(19):3047-8. https://doi.org/10.1093/bioinformatics/btw354.

59. Andersen LF, Solvoll K, Johansson LRK, Salminen I, Aro A, Drevon CA. Evaluation of a food frequency questionnaire with weighed records, fatty acids, and alpha-tocopherol in adipose tissue and serum. Am J Epidemiol 1999;150(1):75-87. https://doi.org/10.1093/oxfordjournals.aje.a009921.

60. Andersen LF, Tomten $H$, Haggarty P, Løvø A, Hustvedt BE. Validation of energy intake estimated from a food frequency questionnaire: a doubly labelled water study. Eur J Clin Nutr. 2003;57(2):279-84. https://doi.org/10.1 038/sj.ejcn.1601519.

61. Carlsen MH, Lillegaard IT, Karlsen A, Blomhoff R, Drevon CA, Andersen LF. Evaluation of energy and dietary intake estimates from a food frequency questionnaire using independent energy expenditure measurement and weighed food records. Nutr J. 2010;9:1-9

62. Andersen LF, Veierød MB, Johansson L, Sakhi A, Solvoll K, Drevon CA. Evaluation of three dietary assessment methods and serum biomarkers as measures of fruit and vegetable intake, using the method of triads. $\mathrm{Br} \mathrm{J}$ Nutr. 2005;93(4):519-27. https://doi.org/10.1079/BJN20041381.

63. Carlsen MH, Karlsen A, Lillegaard ITL, Gran JM, Drevon CA, Blomhoff R, et al Relative validity of fruit and vegetable intake estimated from an FFQ, using carotenoid and flavonoid biomarkers and the method of triads. Br J Nutr. 2011:105(10):1530-8. httos://doi.org/10.1017/S0007114510005246.

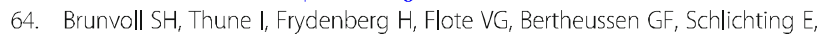
et al. Validation of repeated self-reported n-3 PUFA intake using serum phospholipid fatty acids as a biomarker in breast cancer patients during treatment. Nutr J. 2018:17:1-12.

65. Norwegian Food Safety Authority. Norwegian Food Composition Database 2019 [Internet]. Available from: www.matvaretabellen.no.

66. Johansson I, Hallmans G, Wikman Å, Biessy C, Riboli E, Kaaks R. Validation and calibration of food-frequency questionnaire measurements in the northern Sweden health and disease cohort. Public Health Nutr. 2002;5(3): 487-96. https://doi.org/10.1079/PHN2001315.

67. Holmberg L, Ohlander EM, Byers T, Zack M, Wolk A, Bruce $\AA$, et al. A search for recall Bias in a case-control study of diet and breast Cancer. Int $J$ Epidemiol. 1996;25(2):235-44. https://doi.org/10.1093/ije/25.2.235.

68. Willett W. Nutritional epidemiology. Oxford. New York: Oxford University Press; 2013.

69. World Cancer Research Fund/American Institute for Cancer Research. Diet, nutrition, physical activity and colorectal cancer. Continuous Update Project. 2018. https:/www.wcrforg/sites/default/files/Colorectal-cancer-report. pdf\%0Ahttps:/www.wcrf.org/sites/default/files/Oesophageal-cancer-report. pdf. Accessed 8 June 2021.

70. Shams-White MM, Brockton NT, Mitrou P, Romaguera D, Brown S, Bender A et al. Operationalizing the 2018 World Cancer Research Fund/American Institute for Cancer Research (WCRF/AICR) Cancer prevention recommendations: a standardized scoring system. Nutrients. 2019;11(7): 1572. https://doi.org/10.3390/nu11071572

71. Markussen MS, Veierod MB, Kristiansen AL, Ursin G, Andersen LF. Dietary patterns of women aged 50-69 years and associations with nutrient intake, sociodemographic factors and key risk factors for non-communicable diseases. Public Health Nutr. 2016;19(11):2024-32. https://doi.org/10.1017/ $\$ 1368980015003547$

72. Knudsen MD, Berstad P, Hjartåker A, Gulichsen EH, Hoff G, De Lange T, et al. Lifestyle predictors for non-participation and outcome in the second round of faecal immunochemical test in colorectal cancer screening. Br J Cancer. 2017:117(4):461-9. https://doi.org/10.1038/bjc.2017.189.

73. Folkehelseinstituttet. Reseptregisteret 2012-2016. 2017.

74. WHO Collaborating Centre for Drug Statistics Methodology. Structure and principles https://www.whocc.no/atc/structure_and_principles/. Accessed 28 September 2020

75. WHO Collaborating Centre for Drug Statistics Methodology. ATC/DDD Index $2020 \mathrm{https}: / /$ www.whocc no/atc ddd index/. Accessed 28 September 2020.

76. Cancer Registry of Norway. Cancer in Norway 2018 - Cancer incidence, mortality, survival and prevalence in Norway. Oslo; 2019.

77. Larsen IK, Smastuen M, Johannesen TB, Langmark F, Parkin DM, Bray F, et al. Data quality at the Cancer registry of Norway: an overview of comparability, completeness, validity and timeliness. Eur J Cancer England. 2009:45(7): 1218-31. https://doi.org/10.1016/j.ejca.2008.10.037.

78. Norwegian Institute of Public Health (NIPH). Access to data from the Norwegian Prescription Database. https://www.fhi.no/en/hn/healthregistries/norpd/Access-data-norpd/\#legal-requirements-for-the-disclosureof-data-from-the-norpd. Accessed 19 June 2020.

79. Cancer Registry of Norway. The microbiome as a colorectal cancer screening biomarker. https:/www.kreftregisteret.no/en/Research/Projects/ microbiota-and-lifestyle-in-colorectal-cancer-screeing/. Accessed 29 September 2020

80. Tibshirani R. Regression shrinkage and selection via the lasso. J R Stat Soc Ser B. 1996:58:267-88

81. Cortes C, Vapnik V. Support-vector networks. Mach Learn. 1995

82. Breiman L. Random forests. Mach Learn. 2001:1-122.

83. Haykin S. Neural networks and learning machines. Third Edit. Pearson Prentice Hall; 2009

84. Chen T, Guestrin C. XGBoost: A Scalable Tree Boosting System. Proc 22nd ACM SIGKDD Int Conf Knowl Discov Data Min. 2016;

85. Stark M, Zapf A. Sample size calculation and re-estimation based on the prevalence in a single-arm confirmatory diagnostic accuracy study. Stat Methods Med Res. 2020;

86. Nagata N, Tohya M, Fukuda S, Suda W, Nishijima S, Takeuchi F, et al. Effects of bowel preparation on the human gut microbiome and metabolome. $\mathrm{Sci}$ Rep. 2019;9:1-8.

87. Ahlquist DA, McGill DB, Fleming $J$, Schwartz $S$, Wieand HS, Rubin J, et al Patterns of occult bleeding in asymptomatic colorectal cancer. Cancer. 1989;63(9):1826-30. https://doi.org/10.1002/1097-0142(19900501)63:9<182 6::aid-cncr2820630928>3.0.co:2-o

88. Hannelore D. Diet and the gut microbiome: from hype to hypothesis. $\mathrm{Br} J$ Nutr. 2020:1-24.

89. Berstad P, Løberg M, Larsen IK, Kalager M, Holme $\varnothing$, Botteri E, et al. Long-term lifestyle changes after colorectal cancer screening: randomised controlled trial. Gut. 2015;64(8):1268-76. https:/doi.org/10.1136/gutjnl-2014-307376.

90. Knudsen MD, Hjartåker A, Olsen MKE, Hoff G, De Lange T, Bernklev T, et al. Changes in health behavior 1 year after testing negative at a colorectal cancer screening: a randomized-controlled study. Eur J Cancer Prev. 2018; 27(4):316-22. https://doi.org/10.1097/CEJ.0000000000000328.

\section{Publisher's Note}

Springer Nature remains neutral with regard to jurisdictional claims in published maps and institutional affiliations.

Ready to submit your research? Choose BMC and benefit from:

- fast, convenient online submission

- thorough peer review by experienced researchers in your field

- rapid publication on acceptance

- support for research data, including large and complex data types

- gold Open Access which fosters wider collaboration and increased citations

- maximum visibility for your research: over 100M website views per year

At BMC, research is always in progress.

Learn more biomedcentral.com/submissions 